\title{
Flexible Control and Coupling of Adhesion and Friction of Gecko Setal Array During Sliding
}

\author{
Yu Tian ${ }^{1)^{*}}$, Dashuai Tao $^{1)}$, Noshir Pesika ${ }^{2)}$, Jin Wan ${ }^{1)}$, Yonggang Meng ${ }^{1)}$ and Xiangjun Zhang ${ }^{1)}$ \\ ${ }^{1)}$ State Key Laboratory of Tribology, Tsinghua University \\ Beijing 1000084, China \\ ${ }^{2)}$ Chemical and Bimolecular Engineering Department, Tulane University \\ New Orleans, LA 70118, USA \\ *Corresponding author: tianyu@mail.tsinghua.edu.cn
}

( Manuscript received 21 December 2013; accepted 3 June 2014; published 15 April 2015 ) ( Presented at Technical Session in the $5^{\text {th }}$ World Tribology Congress TORINO September 2013 )

\begin{abstract}
Gecko has remarkable ability to control its adhesion and friction during running to realize a swift climbing or running on vertical walls or upside down ceilings. This ability has received considerable interest of researchers. During the recent decade, significant progress has been made in understanding the mechanism and the biomimetic fabrication of gecko-inspired dry adhesives. While people generally desire a strong and reversible adhesion property, this article described the progress in the investigation of the properties of gecko hierarchical structures, and the corresponding theoretical understandings, and then presented our recent progresses on the flexible controlled adhesion and friction of gecko surface and biomimetic surfaces and a demonstration of a hybrid clamp to ascertain the mechanical property of control principles is also involved. Gecko-inspired adhesives with flexibly controlled adhesion and friction performances have been developed. Macroscopic sliding of those adhesive surfaces with anisotropic hierarchical microscopic structures in a desired direction could be carried out and engage or disengage a flexible control of strong adhesion and friction, and acquire an "intelligent" adhesion.
\end{abstract}

Keywords: gecko setal array, friction, adhesion, anisotropic properties, gecko-inspired adhesives

\section{Introduction}

From ancient ages, gecko has been well-known for its fantastic climbing abilities. For instance, Aristotle $(384 \mathrm{BC}-322 \mathrm{BC})$ has some description of this amazing phenomenon two thousand years ago $[1,2]$. The mechanism of gecko's adhesion and friction has been widely studied. Various hypotheses have been proposed to explain the strong adhesion and friction forces of gecko toe surface, such as electrostatic interaction, vacuum suction, micro-interlocking, and capillary force, which were all eventually disproved by experiments. In the middle of last century, the invention of scanning electron microscopy (SEM) enabled the examination of fine, hierarchical structures of setal lamellar, which is consisted of seta that is approximately $100 \mu \mathrm{m}$ long and a diameter of approximately 4-6 $\mu \mathrm{m}$ and bifurcates into approximately 100-1000 spatulae at the terminal end $[3,4]$. This branched hierarchical structure ensures a close contact between the setae and target surfaces during gecko moving. Due to the discovering of the hierarchical structure of setae, the van der Waals and capillary forces are consider as the two possible major basic interactions between setae and substrates.

Experimental results indicated that van der Waals force is the main contributor to adhesion force, and that the presence of water vapor in the environment may enhance this van der Waals interaction or using capillary force [5-7]. Huber et al. [5]. proposed that the presence of a monolayer or two of water molecules would modify the van der Waals Hamaker constant. Pesika et al [6]. found that the surface hydrophobicity of gecko setae could be changed after exposed to water vapor for a long time. Puthoff et al. discovered that a high humidity could soften the setal $\beta$-keratin, leading to an increase in the adhesion force [7]. The adhesion and friction forces of gecko seta have been tested over the past decade. It has been found that the friction force of an isolated setal array is $\sim 0.37 \mathrm{~N}$, with an apparent contact area of $\sim 1.0$ $\mathrm{mm}^{2}$, whereas that of a single toe is $\sim 4.3 \mathrm{~N}$, with an apparent contact area of $\sim 200 \mathrm{~mm}^{2}$. The maximum friction force of a single seta is approximate $200 \mu \mathrm{N}$, while the adhesive force ranges from $20 \mu \mathrm{N}$ to $40 \mu \mathrm{N}$. Sliding along the curvature of seta is required to obtain 
high friction and adhesion. A single seta spontaneously detaches from a substrate when the setae shaft forms an angle of $30^{\circ}$ to it [8].

Many studies have been devoted to understanding the "frictional adhesion" properties and mechanisms of gecko adhesion in order to mimic its swift movement. Gecko-inspired adhesives have several advantages over those based on vacuum suction, magnetic adsorption, such as small size, flexible control, self-cleaning, and adaptability to rough surfaces $[9,10]$. Therefore, the attachment and detachment mechanisms of gecko feet and the design of gecko-inspired adhesives are of great interest for both theoretical and practical applications. During attachment, gecko presses down its toes on the opposing substrate and followed by sliding toes inward to engage high adhesion and friction forces, while during detachment, the toes roll out from their toe tips and finally detach in the middle place of toes. As predicted in a peel model, by changing the peeling angle of spatulae, the friction and adhesion force originated from the spatulae can be changed by orders of magnitude to realize a strong adhesive contact and easy peel off [11]. When a setal array is sheared against the substrate, the adhesion and friction forces increase gradually as the sliding velocity increases from $5 \mu \mathrm{m} / \mathrm{s}$ to $158 \mu \mathrm{m} / \mathrm{s}$ [12], and the ratio of adhesion to shear force is a constant along the setal shaft arc direction [13]. The adhesion and friction forces of feet during movement have also been measured [14].

However, the friction and adhesion dependence on sliding velocity seems to disagree with the fact that geckos can remain static for a long time on ceilings. Upon tight gripping of toes, the peel angle of the spatulae might decrease, and the contact number of spatulae might also increase. The real process still needs to be resolved. Especially, the function of lamellar structure during the rolling in/out of gecko toes has not been disclosed yet. This study aims to answer these questions and to establish a more comprehensive understanding on the overall mechanism of adhesion and friction control of gecko from microscopic to macroscopic structures.

\section{Experiments}

The experiment was carried out on a home-made apparatus (as sketched in Figs. 1(a) and 1(b)), whose parameters are shown in Table 1. The normal and lateral motions are driven by motors. Two spring cantilevers are used to measure friction and adhesion forces, respectively. Two displacement sensors are used to detect deformation of the springs during test. A glass slide is used as substrate to slide against the setae as shown in Figure 1b. An isolated gecko setal array as shown in Fig. 1(c) is acquired by adhering a gecko toe to a clean glass slide and then sliding quickly. The setal array is cut into small pieces and then glued on the top of steel ball (4 $\mathrm{mm}$ diameter) using $\mathrm{AB}$ glue

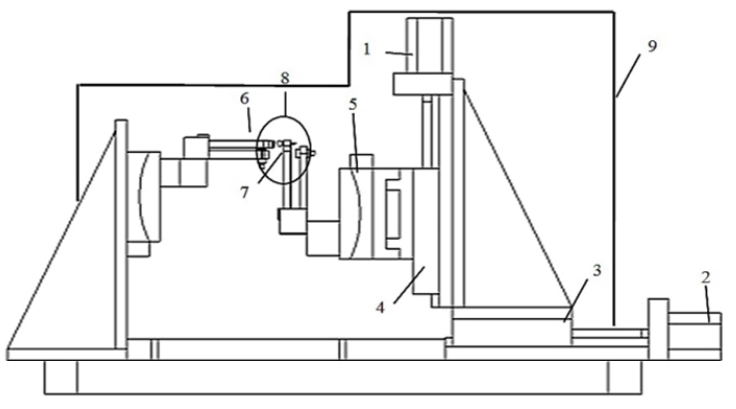

(a)

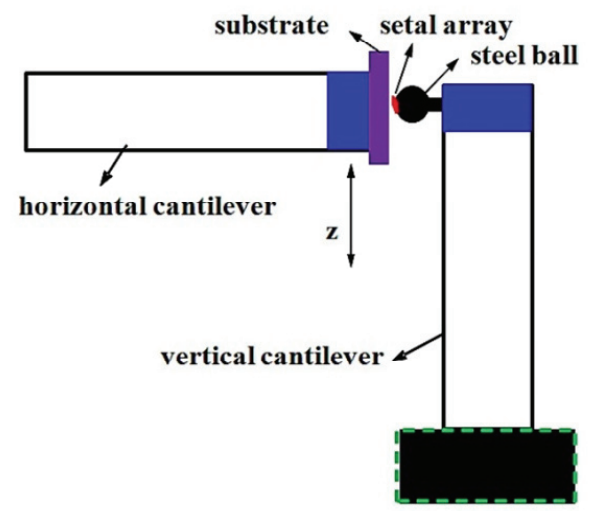

(b)

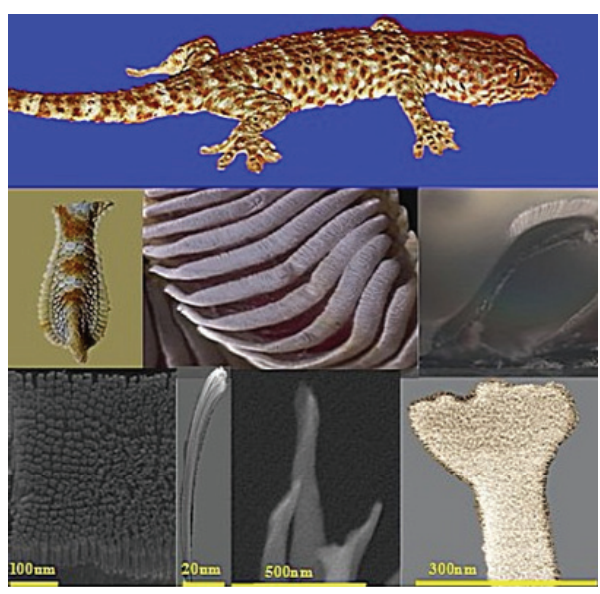

(c)

Fig. 1 The test apparatus. (a) Structure of the apparatus: 1, 2 Motors; 3,4 Guide stage; 5 Angular adjustment part; 6, 7 Spring cantilever; 8 Force measurement part (detailedly shown in (b)). (b) Key parts and samples in the test. (c) The hierarchical structure of gecko surface 
Table 1 Parameters of the test apparatus

\begin{tabular}{lcc}
\hline Force measurement & Horizontal Direction & Vertical Direction \\
\hline Signal transform, $K(\mathrm{mN} / \mathrm{V})$ & 6.96 & 13.40 \\
Sensor output, $s(\mathrm{~V} / \mu \mathrm{m})$ & 0.016 & 0.024 \\
V Range of sensor $(\mathrm{V})$ & $0-10$ & $0-5$ \\
Displacement resolution $(\mathrm{nm})$ & 25 & 10 \\
Cantilever spring, $k(\mathrm{~N} / \mathrm{m})$ & 212 & 168 \\
Force range $(\mathrm{mN})$ & \pm 134 & \pm 34 \\
Force resolution $(\mathrm{mN})$ & 0.05 & 0.002 \\
Force accuracy $(\mathrm{mN})$ & 0.13 & 0.04 \\
\hline
\end{tabular}

(DP420-3M production). The sample is placed at room temperature for 3 hours prior to experiment. Before the sliding test, two cameras are set on the top and side of the sample to adjust the alignment between the setal array surface and the glass substrate. By assembling the setal arrays on different spring cantilevers, the friction and adhesion test can be performed by using different spring constants. While the lateral force along the sliding direction is called friction, the initial applied perpendicular compressive force is called preload. After sliding, the normal force is measured from the deformation of the spring. If the setal array is stretched, the normal force is adhesive force, on the contrary, it is repulsive force.

A hybrid, three-legged clamp is fabricated based on the obtained performances of setal array. Three spring fingers are uniformly distributed and fixed on a cylindrical holder. At the end of fingers, steel balls with setal arrays of approximately $0.33 \times 0.4 \mathrm{~mm}^{2}$ on their tops are attached. Three threads are used to pull and relax the spring fingers by the motion of a small digital motor to simulate the sliding of the setal arrays on a substrate. The sliding distance is designed to exceed 500 $\mu \mathrm{m}$.

Anisotropic hierarchical gecko-inspired adhesives are developed using micro/nano fabrication techniques. Briefly, a $600 \mathrm{~nm}$ oxide layer is grown on a 4 in. silicon wafer using plasma-enhanced chemical vapor deposition. Then positive photoresist is spun onto the wafer. A mask patterned with rectangular structures is used to form the second level structure. After developing, the oxide layer is etched via reactive ion etching with $\mathrm{CF}_{4}$ and the patterned oxide layer forms the final etch mask. Wiping off the remaining photoresist, a new layer of phtotoresist is spun onto the wafer. Then a mask patterned with circular structures is used to form the first level structure. Once the rectangular structure and the circular structures are aligned, the hierarchical structure is formed using deep reactive ion etching. After the mold is fabricated, polydimethylsiloxane (PDMS) is poured onto the mold to make an inverse mold. Before the PDMS curing totally, a certain shear distance is applied to obtain PDMS mold with tilt holes. Finally, polyurethane (PU) is poured onto the PDMS mold and PU-based gecko-inspired surface with hierarchical tilt structure is obtained. A detail is described in $[15,16]$. A sample sized $10 \times 10 \mathrm{~mm}^{2}$ is sheared in gripping and releasing direction to characterize its frictional and adhesive properties.

\section{Results and discussion}

3.1. Frictional and adhesive properties of isolated setal array

Fig. 2 shows typical test results of a setal array with a piece $0.37 \times 0.4 \mathrm{~mm}^{2}$ sliding on a smooth glass slide. The setal array was vertically approached to the glass slide to make a contact at a small preload $L_{0}$. Then lateral to and fro sliding is carried out. Upon the beginning of sliding in the grip in direction (along with the curvature of the setae) $+V$, the normal force $L$ between the setal array and glass slide switched from repulsive force (negative value) $L_{0}$ to attractive force (positive value) $L_{+\mathrm{V}}$. The lateral friction force $F_{+\mathrm{V}}$ continuously increased. In the reverse direction direction (against the setae curvature or grip out direction) $-V$, the friction force $F_{-\mathrm{V}}$ and normal forces $L_{-\mathrm{V}}$ also changed their direction. In this case, the normal force was even higher than its applied preload. The friction force during the reverse sliding is more stable than that sliding in the grip in direction. 


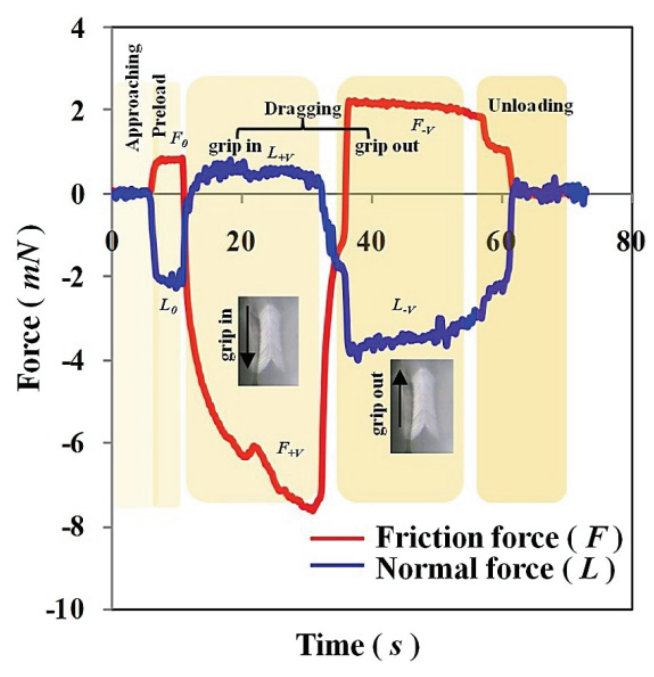

Fig. 2 Typical curves of adhesion (blue) and friction force (red) of gecko setal array during sliding (shearing velocity $100 \mu \mathrm{m} / \mathrm{s}$ )

Sliding the setal array at different preloads, the friction force in the grip in direction $F_{+\mathrm{v}}$ and the normal force $L_{+\mathrm{v}}$ are shown in Figs. 3(a), 3(b). As the increase of the applied preload, the friction force $F_{+\mathrm{v}}$ monotonically increased. The normal force $L_{+_{\mathrm{v}}}$ firstly is adhesive force and the amplitude increased to a maximum value and then decreased, and finally became repulsive force which increased as the further increase of the applied normal load. Figure 3(b) shows more clearly of this process. The slop of the curve is constant at last which indicates the friction of bulks. Sliding the setal array along the grip out or release direction, both the friction force $F_{-\mathrm{v}}$ and the normal force $L_{\mathrm{vv}}$ increased as the increase of the applied load. But there is no adhesive force between the setal array and the opposing glass substrate. Results in Fig. 3(c) show that the friction force is proportional to the normal force with a friction coefficient about 0.6 [17].

$\mu_{i}$ is defined as the friction coefficient when sliding setal array in grip in direction. $\mu_{o}$ is the friction coefficient in grip out direction. Friction coefficient can also imply the efficiency with which normal force generate frictional force. So a parameter $A$ characterizing frictional anisotropic property can be defined,

$$
A=\mu_{i} / \mu_{o}
$$

Eq. (1) gives a criteria to compare anisotropic property of gecko setal arrays or gecko-inspired adhesives.

The experiments of sliding setal array along the grip in direction (with the curvature of the arc curve of setal shafts) or grip out directions (against the curvature of the arc curve of setal shafts) show different frictional and adhesive properties. Along the grip in direction, geckos can gain an attractive force between their toes

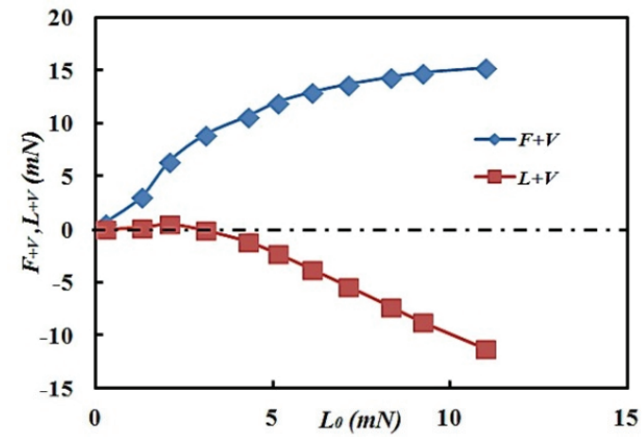

(a)

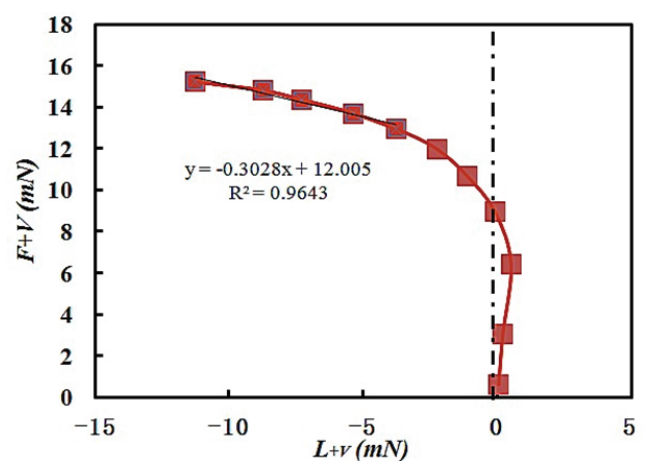

(b)

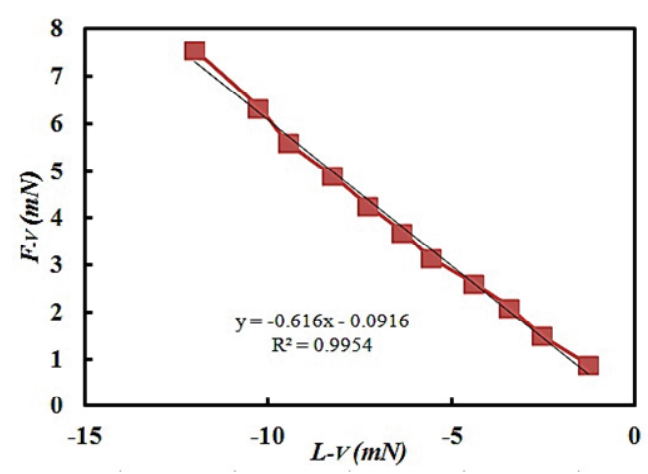

(c)

Fig. 3 Normal force and friction force of gecko setal array during sliding (shearing velocity 100 $\mu \mathrm{m} / \mathrm{s}$ ). (a) Friction force and normal force versus preload in grip in direction; (b) Friction force versus normal force in grip in direction; (c) Friction force versus normal force in grip out direction

and the opposing substrate, while along the grip out direction, only repulsive force is acquired. This strong anisotropic frictional and adhesive properties promised the strong attachment and easy detachment $[1,18]$. As shown in Fig. 4, the setal array during sliding at the both directions is shown in time sequence.

While it seems that the mechanism of the attachment and detachment of gecko setal array has been disclosed, 


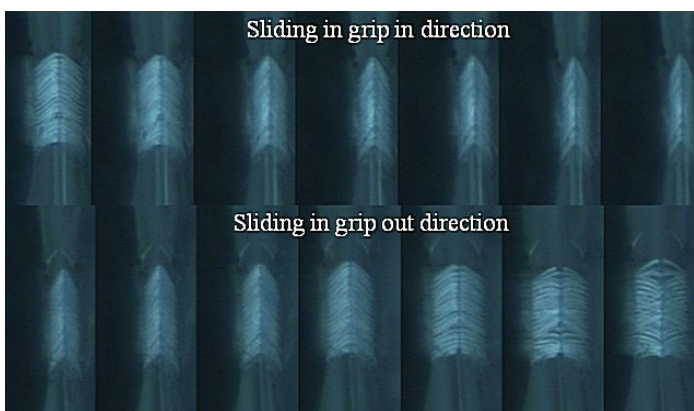

Fig. 4 The images of setal array during sliding in grip in direction (above row) and grip out direction (bottom row)

the experimental study of a single seta by Autumn has found that a sliding is necessary to engage the setal surface and the substrate to achieve a high friction [8]. Thus the sliding distance significantly affects the friction and adhesion. There is no profound study on the sliding distance effect. Considering different preloads leads to different friction and normal forces along the grip in direction, the curves of friction and normal force at different preloads versus the sliding distance are shown in Fig. 5. As shown in those figures, both of the forces initially increases quickly as the increase of the sliding distance, and finally became saturated. The saturated frictional force is higher at higher preloads, while the adhesive force gets a smaller value at a bigger preload. The sliding distance to reach the saturation of forces is about 500-1000 $\mu \mathrm{m}$ [19]. When a gecko climbs on a vertically placed and transparent glass, some images are taken by a high-speed camera on the other side of the glass to capture the gecko toe rolling in/out movements. And the positions of the setal array were determined from the gray pictures to calculate the sliding distance of setal array as shown in Fig. 6, a sliding distance about $200 \mu \mathrm{m}$ has been found during sliding in grip in direction. The discrepancy may be due to the different seta and different substrate and test procedures. The results above are supported by a steel spring, while real gecko setal arrays are standing on their lamellar skin. Whatever the exact value, one can conclude that a few hundreds of sliding distance is necessary for the macroscopic engagement of a setal array surface to a substrate.

Besides the influence of sliding distance on performances of friction and adhesion of gecko setal array when sliding along the grip in direction, above results also show that normal preload could affect stable value of friction and adhesion after sliding. Whether the setal array surface during sliding will be adhesive or repulsive to the substrate highly depends on the preload which is applied through the displacement of a spring supporting the setal array as sketched in Fig. 7(a) [20]. Therefore, the spring should play an important role in the control of adhesion of geckos, which is important for

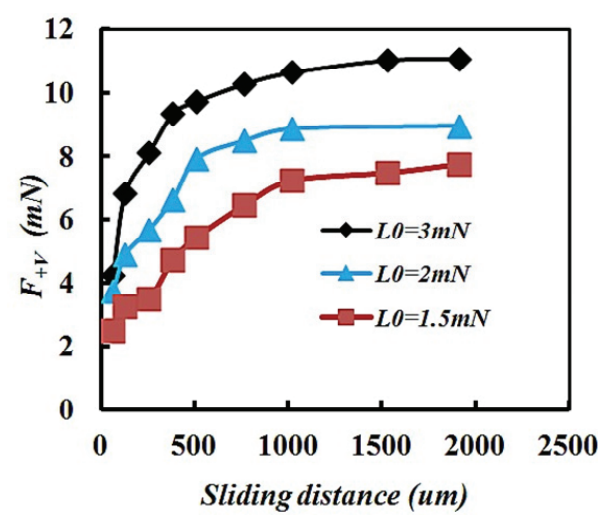

(a)

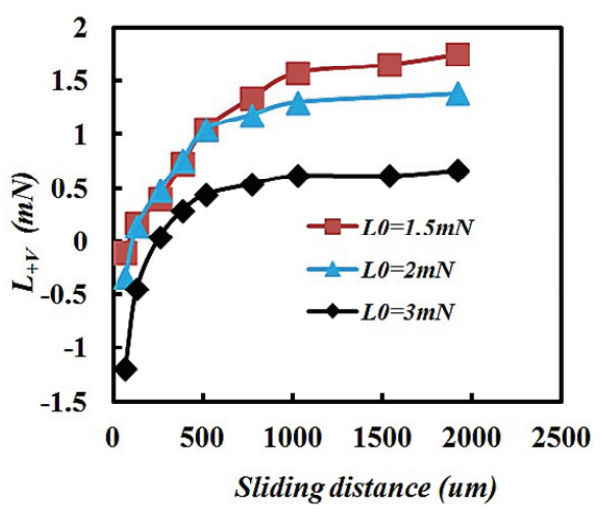

(b)

Fig. 5 Curves of friction (a) and normal force (b) at different preloads versus the sliding distance (shearing velocity $60 \mu \mathrm{m} / \mathrm{s}$; spring constant $196 \mathrm{~N} / \mathrm{m})$

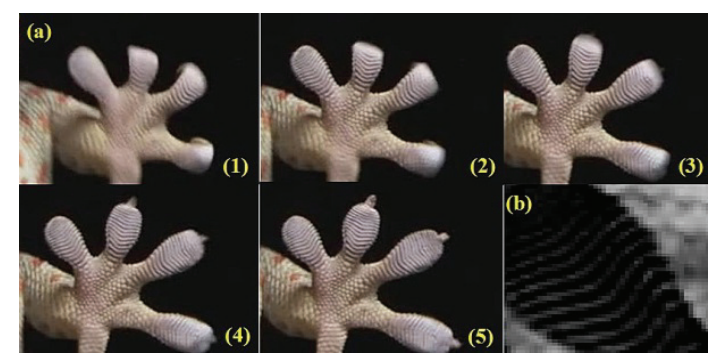

Fig. 6 Images of a gecko toe gripping in to attach to a surface. (a) 1-5 show the gecko toes during gripping in; (b) image of gray difference of big toe in image 2(before shearing) and 5(after shearing)

the design and fabrication of bio-inspired dry and reversible adhesive surfaces. However, most researches are concentrated below the setal level and there is limited discussion on the role of the soft lamellar skin in gecko toe adhesion [21-23]. The lamellar skin on gecko toe acts as a soft spring and sustains most of the normal 


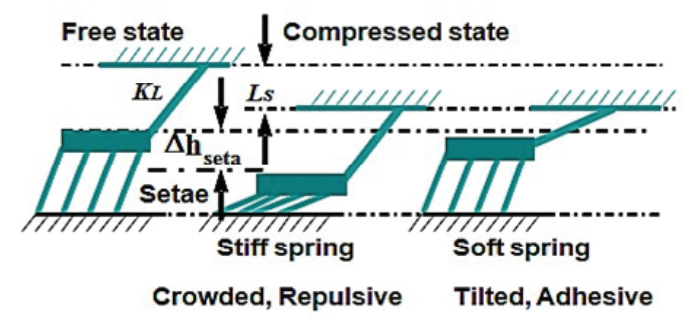

(a)

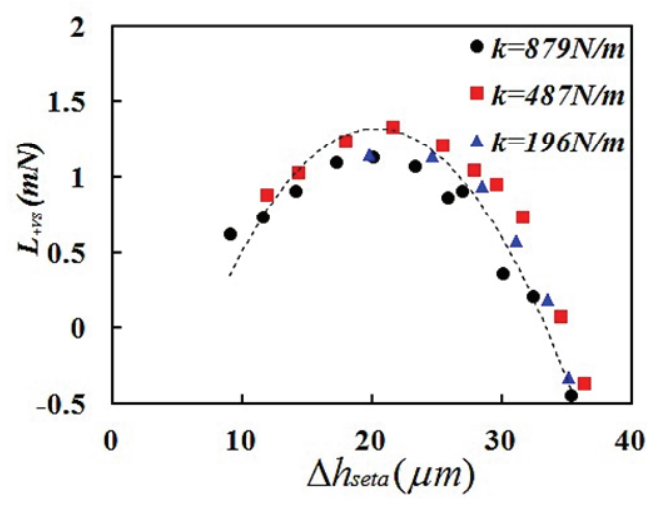

(c)

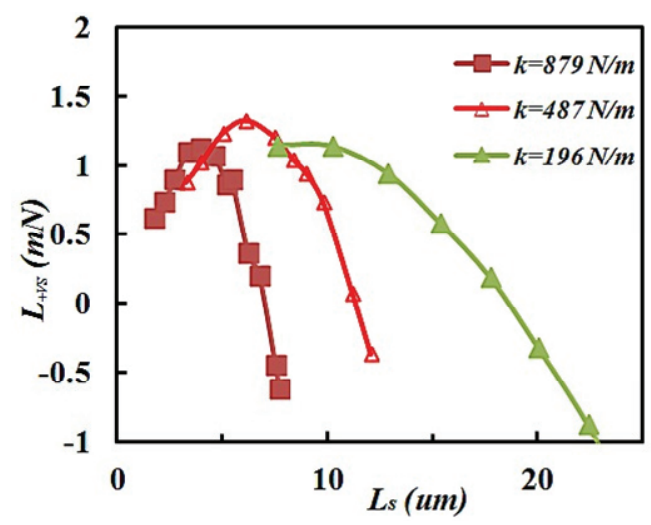

(b)

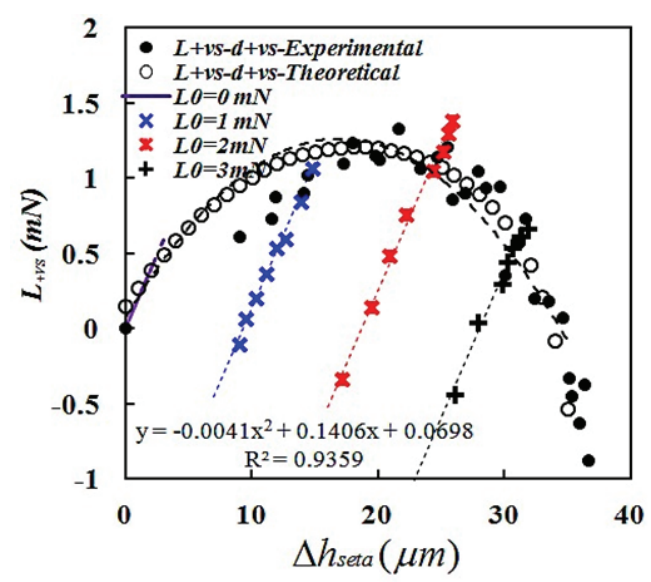

(d)

Fig. 7 Sliding of a setal array at different preload $L_{0}$ with different supporting springs of different spring constant $K_{L}$. (a) sketch of the deformation of the supporting spring $L_{S}$ and the setal array $\Delta h_{\text {seta }}$; (b) stable normal force $L_{+\mathrm{VS}}$ of setal array at different $L_{s}$ and $K_{L}$ (shearing distance $1800 \mu \mathrm{m}$; shearing velocity $60 \mu \mathrm{m} / \mathrm{s}$ ); (c) stable normal force $L_{+\mathrm{VS}}$ versus the deformation of the setal array $\Delta h_{\text {seta }}$ (shearing distance $1800 \mu \mathrm{m}$; shearing velocity 60 $\mu \mathrm{m} / \mathrm{s}$; preload range $1.5-6 \mathrm{mN}$ and step size $0.5 \mathrm{mN}$ ); and (d) the saturated normal force at different preloads (shearing velocity $60 \mu \mathrm{m} / \mathrm{s}$; spring constant $196 \mathrm{~N} / \mathrm{m}$ )

deformation during preloading and maintains a wide range of adhesive rather than repulsive state [20]. Using springs with different stiffness in the sliding measurement of friction and normal force of setal array, results are shown in Fig. 7(b). When changing preload, the displacement of spring will change with it. A lower spring constant could have a wider range of displacement before the adhesive force turns into repulsive force, which means that the adhesive force has a wider safe regime. Nevertheless, there is a one-to-one correspondence between the stable normal force (after a sufficient sliding distance) $L_{+\mathrm{vs}}$ and the stable normal deformation of the setal array $\Delta h_{\text {seta }}$ regardless of the spring constant, which is shown in Fig. 7(c). The formula in Fig. 7(d) fitted one of the curve (when $k$ is 196 N/m) in Fig. 7(c). When the spring constant (i.e. the slope of the fitted lines) is fixed, the value of normal force after sliding sufficiently can be figured out if the preload is decided. And with different preload, the maximum normal force can be adjusted. Thus a conclusion can be drawn that the normal force can be repulsive if the preload is larger enough. As discussed above, gecko can adjust the amount of adhesive and frictional forces either by changing sliding distance or preload, which is quite significant for gecko to climb on various surfaces.

The lamellar skin of gecko and its properties (i.e. stiffness) can significantly contribute to the efficient and reliable control of the attachment and detachment of setal arrays during gecko walking on surfaces [19]. The sequential engagement and peeling off of setal array are responsible for the reliable gecko adhesion and friction control. We proposed that this soft spring supporting pillar structure should be adopted in future bio-inspired 


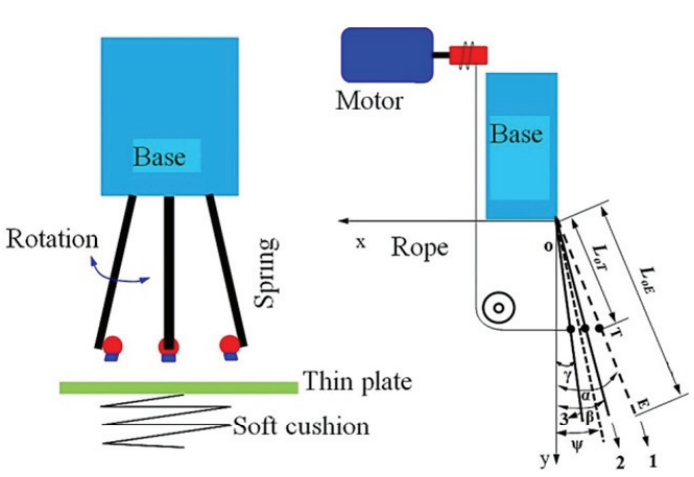

(a)

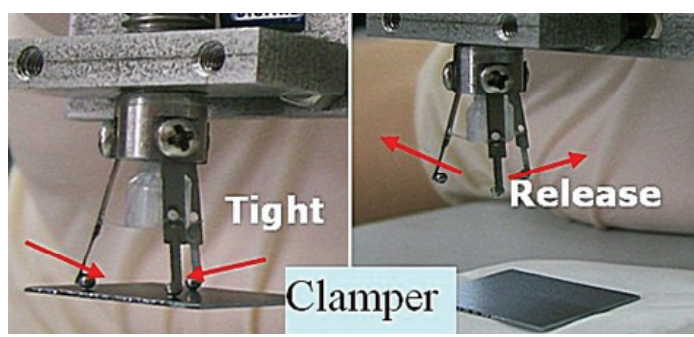

(b)

Fig. 8 Sketch of the three-legged clamp (a) and (b) schematic of working process

adhesives design and fabrication. A lamellar skin/spring-like structure should be incorporated into future generations of gecko-inspired adhesives to provide more control on adhesion and stronger adaptability to surfaces.

\subsection{Design of the hybrid three-legged clamp}

Based on the results above, a hybrid three-legged spring/setae clamp utilizing the anisotropic property of gecko toes has been developed to transfer a horizontally placed silicon wafer. Isolated setal arrays are glued on steel balls which is located at the end of metal legs described in details in [20]. The motion of the metal legs along the grip in direction could acquire adhesive force and will lift up the silicon wafer, while in the opposite direction the silicon wafer will be released, as shown in Fig. 8. The preload and the sliding distance should be optimized to ensure the stability of the clamp. This indicates the importance of integration and optimization of micro or nanoscale structures as well as the incorporation of their unique, size-dependent properties into functional macroscale devices.

\subsection{Frictional and adhesive properties of anisotropic} gecko-inspired adhesives

Finally, since there has been wide interest in fabricating dry adhesives mimicking the gecko adhesive surface, which offers some advantages over

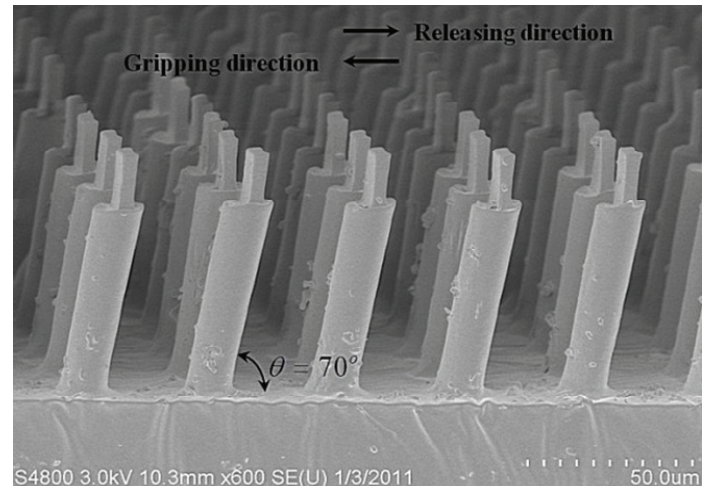

(a)
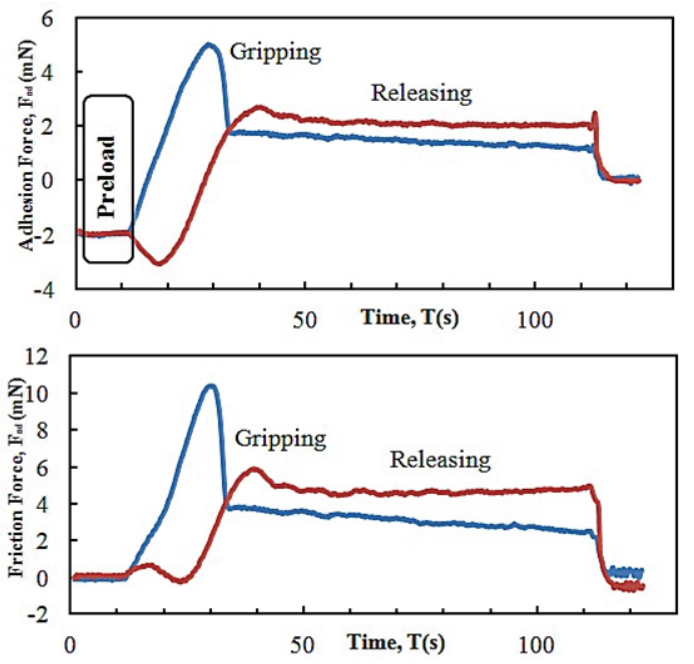

(b)

Fig. 9 Hierarchical structure of gecko-inspired adhesives. (a) gecko-inspired adhesives composed of polyurethane with pillars at $70^{\circ}$ tilt angle; (b) Dynamic measurements of the adhesion forces (above) and friction forces (blow) generated by the gecko-inspired adhesive sample when sheared in the gripping and releasing direction (Dragging velocity: 10 $\mu \mathrm{m} / \mathrm{s}$; Preload: $2 \mathrm{mN}$ )

conventional pressure sensitive adhesives, especially, its anisotropic adhesion properties: the adhesive pads (spatulae) stick strongly when sheared in one direction but are non-adherent when sheared in the opposite direction as shown in Fig. 2. This anisotropy property is attributed to the complex topography of the array of fine tilted and curved columnar structures (setae) that supporting the spatulae. Using a scalable method, relying on conventional and unconventional techniques, incorporating tilt in the fabrication of synthetic polymer-based dry adhesives can mimic the gecko setae with anisotropic adhesion properties as shown in Figure 
3. The anisotropic adhesion and friction properties of samples with tilt angle shown in Fig. 9(a) have been tested. Typical results are shown in Fig. 9(b). When a $70^{\circ}$ tilt sample was actuated in the grip in direction (consistent with the tilt), a maximal adhesion force of 5 $\mathrm{mN}$ and a maximal friction force of $10 \mathrm{mN}$ were obtained. As a contrast, when the dry adhesive was actuated in the releasing direction (against the tilt), an initial repulsive normal force and negligible friction were measured. Samples with various tilt angles are also fabricated and tested $[15,16]$. Consistent with the peel zone model, samples with lower tilt angles yielded larger adhesion forces.

\section{Conclusions}

(1) Isolated setal array has been dragged on a home-made apparatus to characterize its frictional and adhesive properties. The setal array shows an obvious difference in its deformation at different sliding directions, thus revealing strongly anisotropic friction and adhesion. A criteria is proposed to compared the frictional anisotropic property of gecko setal arrays or gecko-inspired adhesives.

(2) Friction and adhesion are strongly affected by preload, sliding distance, and supporting spring constant. A two-level spring model is established to analyze the influence of those factors which could interpret the reason why gecko can stay on walls or even ceils safely. The relationship between deformation of setal array and adhesive force is also revealed.

(3) Based on the experimental results of gecko setal array, a hybrid clamper has been established using the coupled adhesion and friction of setae. This apparatus can generate adhesion force or repulsive force between gecko setal array and silicon wafer which could grip or release the wafer. A novel gecko-inspired adhesive with hierarchical structures is developed and the adhesive surface shows anisotropic property when sliding in opposite directions.

\section{Acknowledgements}

This work was sponsored by the National Basic Research Program of China, Grant No. 2011CB707603, the National Natural Science Foundation of China, Grant No. 51175281 and the National Natural Science Foundation of China with Grant No.51323006.

\section{References}

[1] Autumn, K. and Peattie, A. M., "Mechanisms of Adhesion in Geckos," Integrative and Comparative Biology, 42, 6, 2002, 1081-1090.
[2] Autumn, K., "How Gecko Toes Stick: The Powerful, Fantastic Adhesive Used by Geckos is Made of Nanoscale Hairs that Engage Tiny Forces, Inspiring Envy among Human Imitators," American Scientist, 94, 2, 2006, 124-132.

[3] Ruibal, R. and Ernst, V., "The Structure of the Digital Setae of Lizards," Journal of Morphology, 117, 3, 1965, 271-293.

[4] Russell, A. P., Bauer, A. M. and Laroiya, R., "Morphological Correlates of the Secondarily Symmetrical Pes of Gekkotan Lizards," Journal of Zoology, 241, 4, 1997, 767-790.

[5] Huber, G., Mantz, H., Spolenak, R., Mecke, K., Jacobs, K., Gorb, S. N. and Arzt, E., "Evidence for Capillarity Contributions to Gecko Adhesion from Single Spatula Nanomechanical Measurements," Proceedings of the National Academy of Sciences of the United States of America, 102, 45, 2005, 16293-16296.

[6] Pesika, N. S., Zeng, H., Kristiansen, K., Zhao, B., Tian, Y., Autumn, K. and Israelachvili, J., "Gecko Adhesion Pad: a Smart Surface?," Journal of Physics: Condensed Matter, 21, 46, 2009, 464132.

[7] Puthoff, J. B., Prowse, M. S., Wilkinson, M. and Autumn, K., "Changes in Materials Properties Explain the Effects of Humidity on Gecko Adhesion," The Journal of Experimental Biology, 213, 2010, 3699-3704.

[8] Autumn, K., Liang, Y. A., Hsieh, S. T., Zesch, W., Chan, W. P., Kenny, T. W., Fearing, R. and Full, R. J., "Adhesive Force of a Single Gecko Foot-hair," Nature, 405, 2000, 681-685.

[9] Cutkosky, M. R., "Gecko-like Robot Scampers up the Wall," New Scientist, 2552, 2006, 29-33.

[10] Menon, C., Murphy, M. and Sitti, M., "Gecko Inspired Surface Climbing Robots," Proceedings of the IEEE International Conference on Robotics and Biomimetics, Shenyang, China, 2004, 431-436.

[11] Tian, Y., Pesika, N., Zeng, H., Rosenberg, K., Zhao, B., McGuiggan, P., Autumn, K. and Israelachvili, J., "Adhesion and Friction in Gecko Toe Attachment and Detachment," Proceedings of the National Academy of Sciences, 103, 51, 2006, 19320-19325.

[12] Yamaguchi, T., Gravish, N., Autumn, K. and Creton, C., "Microscopic Modeling of the Dynamics of Frictional Adhesion in the Gecko Attachment System," The Journal of Physical Chemistry B, 113, 12, 2009, 3622-3628.

[13] Autumn, K., Dittmore, A., Santos, D., Spenko, M. and Cutkosky, M., "Frictional Adhesion: A New Angle on Gecko Attachment," Journal of Experimental Biology, 209, 2006, 3569-3579.

[14] Irschick, D. J., Vanhooydonck, B., Herrel, A. and Andronescu, A., "Effects of Loading and Size on Maximum Power Output and Gait Characteristics 
in Geckos," Journal of Experimental Biology, 206, 2003, 3923-3934.

[15] Jin, K., Tian, Y., Erickson, J. S., Puthoff, J., Autumn, K. and Pesika, N., "Design and Fabrication of Gecko-inspired Adhesives," Langmuir, 28, 2012, 5737-5742.

[16] Jin, K., Cremaldi, J. C., Erickson, J. S., Tian, Y., Israelachvili, J. N. and Pesika, N. S., "Biomimetic Bidirectional Switchable Adhesive Inspired by the Gecko," Advanced Functional Materials, 24, 5, 2014, 574-579.

[17] Jin, W., Yu, T., Ming, Z., Xiang-Jun, Z. and Yong-Gang, M., "Experimental Research of Load Effect on the Anisotropic Friction Behaviors of Gecko Seta Array," Acta Physico-Chimica. Sinica., 61, 1, 2012, 016202 (in Chinese) .

[18] Zhao, B., Pesika, N., Rosenberg, K., Tian, Y., Zeng, H., McGuiggan, P., Autumn, K. and Israelachvili, J., "Adhesion and Friction Force Coupling of Gecko Setal Arrays: Implications for Structured Adhesive Surfaces," Langmuir, 24, 4, 2008, 1517-1524.
[19] Wan, J., "Experimental Study on the Adhesion and Friction Mechanism of Gecko Setae Array," Master Thesis, Tsinghua University, Beijing, China, 2012 (in Chinese).

[20] Tian, Y., Wan, J., Pesika, N. and Zhou, M., "Bridging Nanocontacts to Macroscale Gecko Adhesion by Sliding Soft Lamellar Skin Supported Setal Array," Scientific Reports , 3, 2013, 1382.

[21] Zhou, M., Liu, K., Wan, J., Li, X., Jiang, K., Zeng, H. B., Zhang, X. J., Meng, Y. G., Wen, S. Z., Zhu, H. W. and Tian, Y., "Anisotropic Interfacial Friction of Inclined Multiwall Carbon Nanotube Array Surface," Carbon, 50, 15, 2012, 5372-5379.

[22] Sitti, M. and Fearing, R. S., "Synthetic Gecko Foot-hair Micro/nano-structures as Dry Adhesives," Journal of Adhesion Science and Technology, 17, 8, 2003, 1055-1073.

[23] Qu, L. T., Dai, L. M., Stone, M., Xia, Z. and Wang, Z. L., "Carbon Nanotube Aarrays with Strong Shear Binding-on and Easy Normal Lifting-off," Science, 322, 5899, 2008, 238-241. 